\title{
Perspectives on the draft ICH M10 guidance: an interview with Kelly Dong
}

\author{
Kelly Dong ${ }^{*, 1}$ \\ ${ }^{1}$ United-Power Pharma Tech Co., Ltd, Zhong-Guan-Chun Life Science Park, Beijing 102206, PR China \\ *Author for correspondence: kellydong@up-pharma.com
}

Kelly Dong, PhD, Chief Executive Officer, United-Power Pharma Tech Co., Ltd: Kelly Dong obtained her PhD degree from McGill University, Canada. Kelly has nearly 25 years of multinational industry experience working for pharmaceutical companies and CROs in Canada, the UK and China. Her scientific expertise encompasses drug metabolism and pharmacokinetics (DMPK) in drug discovery and regulated bioanalysis for preclinical and clinical development. After 20-year overseas experience, she joined GlaxoSmithKline R\&D China in August 2009. She was the Director of DMPK for CNS drug discovery and Head of Bioanalysis, Immunogenicity and Biomarkers, overseeing more than 40 preclinical and clinical studies across different therapeutic areas. She joined United-Power Pharma as the Chief Executive Officer in February 2018. She is also a research fellow at the National Engineering Research Center of Protein Drugs. She is one of the founders and a steering committee member of China Bioanalysis Forum. She is also an active contributor to the scientific community, with numerous scientific publications, invited presentations and organizing scientific conferences.

This interview was conducted by Sankeetha Nadarajah, Managing Commissioning Editor of Bioanalysis.

Accepted for publication: 11 October 2019; Published online: 24 October 2019

Keywords: bioanalytical method validation • China Bioanalysis Forum • global harmonization • ICH M10 feedback - regulatory landscape

\section{Can you provide a brief overview \& recent developments of the Chinese Regulatory landscape?}

The Chinese pharmaceutical industry has been undergoing rapid growth in the past decade. A number of regulatory reforms have been in place to accommodate the paradigm changes. The changes include the strong emphasis on data quality and standards through enforced regular inspections, implementation of a 60-day clinical trial filing policy, significantly increased numbers of reviewers and a much reduced new drug application (NDA) approval process. It also became possible to initiate Phase I and II clinical trials in China parallel to global development. The centralized government procurement program, exemplified by recent $4+7$ cities procurement scheme, could warrant the sales volume in exchange of a much reduced profit margin for the listed products. This will inevitably speed up the consolidation of Chinese generic pharmaceutical industry and also send a strong signal for innovation. As a result of these changes, there was a 75\% increase in innovative drug applications in 2018 as compared with 2 years ago. An increasing number of Chinese pharmaceutical companies are expanding their footprints to North America and Europe while aiming for global submission. China also became an official member of the ICH in June 2017, which marked a new era of globalization. China has also been actively participating in the ICH guideline consultation, including M10 guideline.

\section{How will the ICH M10 guidance be different to currently available Bioanalytical Method Validation guidance used in China?}

The most relevant guideline in China for regulated bioanalytical studies refers to the Guideline for Bioanalytical Method Validation (BMV) in Chinese Pharmacopeia [1]. The major components for BMV are very similar between the Chinese Pharmacopeia compared with ICH M10 draft guideline, although a few minor differences can be noted, including the test article concentration for selectivity assessment in ligand binding assays (LBA; LLOQ and ULOQ vs LLOQ and high QC) and the requirement on whether the QC should be freshly prepared for accuracy 
and precision assessments. In addition, the Chinese Pharmacopeia does not clearly specify the number of runs needed for dilution integrity or a temperature bracketing approach for stability assessments. Interestingly, both the Chinese Pharmacopeia and M10 draft have specified a need for 100\% chromatograms for bioequivalence studies, which inevitably requires additional effort for documentation preparation.

\section{Can you explain the process of industry feedback collation in China with regards to the draft ICH M10?}

China has two representatives from local regulatory agencies who participate in the global ICH M10 discussion. Industry feedback has been collected mainly through two different channels, particularly via the R\&D-Based Pharmaceutical Association Committee and the China Bioanalysis Forum (CBF). R\&D-Based Pharmaceutical Association Committee, a member of the International Federation of Pharmaceutical Manufacturers \& Associations, comprises of members from multiple global pharmaceutical companies with $\mathrm{R} \& \mathrm{D}$ activities in China. It is appreciated that a majority of pharmaceutical companies in China have rather limited in-house bioanalytical expertise. As a nonprofitable organization, the CBF is the most recognized bioanalytical-focused group in China. CBF led the feedback collection on the ICH M10 draft guideline from its expert committee members and other local experts in the field. The summarized feedback were communicated during the European Bioanalysis Forum (EBF) spring meeting in May 2019 and American Association of Pharmaceutical Scientists (AAPS) ICH M10 workshop in June 2019. The collective feedback from CBF, also including some practical considerations reflecting region-specific situations, for example, challenges in sample import/export, has been delivered to local regulatory.

\section{How easily can laboratories adapt to implement the ICH M10 guidance?}

A majority of the requirements for bioanalytical method validation are similar between the ICH M10 draft guideline and the currently used Guideline for Bioanalytical Method Validation in China Pharmacopeia [1]. It is the intention of local regulatory agencies to align the ICH guideline with global standards. Interestingly, most of the well-established laboratories for regulated bioanalysis in China reside in several bioanalytical CROs which have experience supporting regulatory submission in China, North America and Europe. The implementation of the ICH M10 guideline could further standardize the approach in the industry globally and will be much welcomed by local bioanalytical community.

\section{Was the draft what you expected from a harmonized consensus guidance?}

In general, the ICH M10 draft guideline is a well-prepared document that provides clear instructions and necessary details for the regulatory recommendations. Most of the recommended approaches are in line with the industry's current practices, although there are still some areas that could be further clarified and streamlined with scientific justification, such as the recommendation for dilution linearity assessment and cross validation. In addition, the ICH M10 guideline will likely be adopted by many countries across continents. Some region-specific situation may have an impact on its implementation, such as cross validation sample preparation and shipment. In addition, the acquisition of human matrices from disease patients has always been a practical and ethical challenge during method development and validation.

\section{Disclaimer}

The opinions expressed in this interview are those of the interviewee and do not necessarily reflect the views of Newlands Press Ltd.

Financial \& competing interests disclosure

The author has no relevant affiliations or financial involvement with any organization or entity with a financial interest in or financial conflict with the subject matter or materials discussed in the manuscript. This includes employment, consultancies, honoraria, stock ownership or options, expert testimony, grants or patents received or pending, or royalties.

No writing assistance was utilized in the production of this manuscript.

\section{References}

1. Chinese Pharmacopeia “2019 Guideline for Bioanalytical Method Validation” (2015). 